\title{
Underwater acoustic modem with streaming video capabilities
}

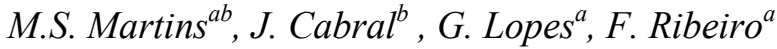 \\ ${ }^{a}$ Algoritmi Center, University of Minho, Campus of \\ Azurém, Guimarães, Portugal \\ ${ }^{b}$ MEMS-UMinho, University of Minho, Campus of \\ Azurém, Guimarães, Portugal \\ mmartins@dei.uminho.pt
}

\begin{abstract}
Oceans have shown tremendous importance and impact on our lives. Thus the need for monitoring and protecting the oceans has grown exponentially in recent years. On the other hand, oceans have economical and industrial potential in areas such as pharmaceutical, oil, minerals and biodiversity. This demand is increasing and the need for high data rate and nearreal-time communications between submerged agents became of paramount importance. Among the needs for underwater communications, streaming video (e.g. for inspecting risers or hydrothermal vents) can be seen as the top challenge, which when solved will make all the other applications possible. Presently, the only reliable approach for underwater video streaming relies on wired connections or tethers (e.g. from ROVs to the surface) which presents severe operational constraints that makes acoustic links together with AUVs and sensor networks strongly appealing. Using new polymer-based acoustic transducers, which in very recent works have shown to have bandwidth and power efficiency much higher than the usual ceramics, this article proposes the development of a reprogrammable acoustic modem for operating in underwater communications with video streaming capabilities. The results have shown a maximum data-rate of $1 \mathrm{Mbps}$ with a simple modulation scheme such as $\mathrm{OOK}$, at a distance of $20 \mathrm{~m}$.
\end{abstract}

Keywords- Underwater communication; streaming video; acoustic communications; ultrassond transducers.

\section{INTRODUCTION}

The demand for ocean exploitation has generated the need for reliable and operational underwater acoustic communications (UACs) which allows for high data-rate pointto-point (P2P) links and for underwater acoustic networks (UAN) implementation [1]. On the other hand, technology improvements in UACs and UANs are nowadays inseparable of ocean sustainable, "blue", exploitation [2]. The emergence of the "blue" paradigm becomes decisive for technology improvements in new and old needs as underwater sensor networks, divers and submarine communications, robotics and Autonomous Underwater Vehicle (AUVs) navigation and control. Those applications are essential to the progress in different areas [2] [3], such as: environmental protection, water conditions and climate change; ocean and marine life; ocean mapping and assisted navigation; disaster prevention and tsunamis detection; military applications. Therefore, it is imperative to find reliable solutions, able to fulfil all these needs.
There are three main methods to communicate through water: acoustic, radio frequency and optical devices [4]. Radio frequency and Optical systems are mainly limited by the high level of absorption in water [5] [6]. As a result, acoustic communication systems are the preferential form for wireless underwater communications, since they present lower attenuation in water [7]. However, reliable UAC is still a great challenge due to environmental effects distorting the signal between emitter and receiver. Among the most impairing effects are: channel multipath, channel time-variability and the limited bandwidth and power efficiency of the mostly used ceramic piezoelectric transducers. In what concerns UANs most of recent work is focused on developing appropriate protocols for dealing with large propagation delays, low datarate and temporary communication failures between nodes [1] [3]. In what concerns P2P communications, in the last two decades, most of the research was focused on the design of adaptive channel equalizers that attempt to compensate channel multipath and track ocean variability constantly minimizing its effect [8] [9]. A more recent approach addresses the use of more bandwidth efficient modulation, as is the case of OFDM [10] [11] and multiple transmitters and coding [12]. It should be noted that in UAC the appropriate performance of a system, in a given time and location, does not guarantee a similar performance at different times and locations; this motivates the emergence of reconfigurable (hardware) systems [13] able to adapt to a special condition.

Despite all the efforts, UACs are far from being efficient and reliable, and recent works are now also focused on using higher frequencies (above $100 \mathrm{kHz}$ ) and on the development of new transducers with larger bandwidths. In [14] the authors presented an acoustic Field Programmable Gate Array (FPGA) based modem, operating at frequencies between 0.1 and $1 \mathrm{MHz}$, for distances ranging between $50 \mathrm{~m}$ and $100 \mathrm{~m}$. Using a Binary Phase Shift Keying modulation with $800 \mathrm{kHz}$ carrier frequency, the system archived a $80 \mathrm{kbps}$ data rate. In [15] [16] [17] a polymer-based directional transducer was developed with a bandwidth between 0.5 and $1 \mathrm{MHz}$, and poolexperimental results have shown a data-rate of $1 \mathrm{Mbps}$ with a simple modulation scheme such as OOK at a distance of $20 \mathrm{~m}$.

The first step for any exploitation, research, monitoring and surveillance (e.g. environmental) is to set the communications infrastructure [4]. Broadband wireless communications is a first requirement for a wide range of applications like: sensor 
networks, single and/or multiple AUVs command and control [18] or video and sensor-data streaming from submerged platforms. However, for achieving a reliable high data-rate streaming, broadband acoustic transducers are required [7] and in addition, reconfigurable modems able to adapt their modulation and equalization schemes to the environmental conditions are an asset. With those in hands, the next logical step is to develop/implement network protocols which are able to deal with the underwater channel specificities and which allow to connect submerged sensors, vehicles, and other devices to the surface in a reliable manner.

\section{DEVELOPMENT OF BROADBAND ULTRASONIC TRANSDUCERS}

The transducer (either projector or hydrophone) geometry and design are directly associated to the frequency band, beam pattern, acoustic power and operation mode [19]. The ultrasonic transducer can be optimized to project, receive or both, but the definition of the operating frequency for a hydrophone or a projector have different meanings. Projectors usually take leverage of the resonance effect, using only drive signals at the resonance frequencies, where the highest acoustic pressure is delivered to the output. Hydrophones, on the other hand, are usually used below resonance frequencies over a much wider frequency band [20]. Hydrophones and projectors can be planar, cylindrical or spherical, mounted with only one transducer or in structures like arrays or matrices using up to thousands of transducers [19].

However, for communications using digital modulations, the transducer geometry must obey different rules. The transducer cannot be optimized to resonance mode but to perform wideband at high frequencies (from $\mathrm{kHz}$ to $\mathrm{MHz}$ range) with low power consumption. To achieve these requirements the transducer has to present reduced damping and acoustic impedance in order to minimize the signal deformation when converted from electric to acoustic.

Usually the transducers are fabricated with ceramic piezoelectric materials, since normally they have higher piezoelectric coefficients. They also have high acoustic impedances. Using polymer or copolymer piezoelectric composites it is possible to reduce the transducer acoustic impedance, ensuring better acoustic energy transference between the transducer and the medium. This improvement means that the internal damping effect is reduced resulting in an acoustic signal closer to the electric signal applied to the transducer. This type of material also requires less energy for working than the ceramics family. Nevertheless, polymers normally present a low acoustic power at the output. But using composites it is possible to integrate a few advantages of polymer and ceramic materials in the same material, with the aim of finding an advantageous relationship between frequency band, acoustic power and energy consumption.

\section{A. Basics of Piezoelectric Ultrasonic Transducers}

Commonly, the piezoelectric ultrasonic transducers operate in the 33 mode, meaning that the deformation occurs along the polarization axis with the excitation electric field point in the same direction. The material free displacement, is given by [4]:

$$
\xi=d_{33} v n
$$

where $\xi$ is the free displacement, $v$ is the applied voltage, $n$ is the number of layers and $d_{33}$ is the coupling coefficient in the thickness direction. The deformation creates a pressure wave in the medium, whose force amplitude can be obtained by [5]:

$$
F_{W}=A_{p} 2 \pi c \rho f \xi
$$

where $c$ is the sound speed, $\rho$ is the material density, $A_{p}$ is the area of the piezoelectric element and $f$ is the signal wave frequency. Despite this increase in strain, the intensity of the produced force does not change [4]. The maximum force that the piezoelectric can apply to a medium is obtained by [4]:

$$
F_{T}=d_{33} \frac{A_{p}}{S_{33}^{E} t_{p}} v
$$

where $S^{E}{ }_{33}$ is the elastic compliance coefficient and $t_{p}$ is the thickness of a single layer.

To ensure the transducer the proper functioning, the force that the transducer $\left(F_{T}\right)$ can apply to the medium must be greater than the generated acoustic wave force $\left(F_{W}\right)$, otherwise the piezoelectric material displacement will be deformed generating acoustic waves with low amplitude and noise. This condition allows for the calculation of the layer thickness for a specific frequency and material. Through equations 1 and 3 it is possible to obtain:

$$
t_{p} \leq \frac{1}{n 2 \pi c \rho S_{33}^{E} f}
$$

Another important aspect is related to the transducer acoustic impedance. The sound wave created inside the transducer reflects, in part, at the boundary established by different densities and bulk modulus B of the transducers and the medium. This reflection creates deformations on the acoustic signal transmitted to the medium, which can be calculated by Eq. 5:

$$
S_{\text {out }}(t)=T_{w}\left(S_{\text {in }}(t)+L R_{w} S_{\text {in }}\left(t+D_{p}\right)\right)
$$

Here, $S_{\text {out }}$ is the sound wave output as function of time $t, S_{\text {in }}$ is the sound wave as function of time created inside the active element, $T_{w}$ is the transmitted sound wave intensity percentage, $R_{w}$ is the reflected sound wave intensity percentage, $L$ is the internal energy loss and $D p$ is the delay of the reflected sound wave, introduced by the active element thickness.

\section{B. Piezoelectric Emitter Transducers Fabrication}

Before the transducer design and fabrication it is necessary to define the transducer characteristics and parameters. Considering that the transducer will be used in underwater communications using digital modulations, the transducer has to operate with frequencies up to $1 \mathrm{MHz}$ with wideband behaviour (100 kHz to $1 \mathrm{MHz}$ ) and low power consumption. For a wideband response, the piezoelectric material as to present an acoustic impedance near to the medium acoustic impedance (around $1.5 \times 10^{6} \mathrm{~kg} / \mathrm{m}^{2} \mathrm{~s}$ ), the nearest better will be the energy transference from the transducer to the medium, 
reducing the signal distortion and attenuation. Therefore, it was selected the PVDF polymer material in piston configuration transducer, since it is an interesting material due to the low acoustic impedance $\left(3.3 \times 10^{6} \mathrm{~kg} / \mathrm{m}^{2} \mathrm{~s}\right)$ and price [9] [10]. Another advantage of the PVDF is the power consumption in comparison to the common Ceramic materials such as Lead Zirconate Titanate (PZT), Lead Titanate (PT), Lead Magnesium Niobate (PMN) and Lead Zinc Niobate (PZN) [6]. However to reduce the power consumption, the transducer will be designed to present a directional beam pattern, focusing the acoustic energy in the receiver. For a divergence angle of $4.3^{\circ}$ and considering piston configuration the transducer has to have $20 \mathrm{~mm}$ of diameter.

To operate up to $1 \mathrm{MHz}$ the maximum thickness cannot exceed $225 \mu \mathrm{m}$ as it was presented in Eq. 4, and to increase the pressure wave output it was selected a multilayer structure with 2 layers of $110 \mu \mathrm{m}$. Table I shows the main physical properties of PVDF to the proposed application, according to equations 1 to 5 :

TABLE I: MECHANICAL AND PIEZOELECTRIC CHARACTERISTICS OF PVDF [9].

\begin{tabular}{|l|c|}
\hline \multicolumn{1}{|c|}{ Physical Property } & PVDF 2x110 $\boldsymbol{\mu m}$ \\
\hline Thickness $(\mathrm{m})$ & $1,10 \mathrm{E}-04$ \\
\hline Resonance Frequency $(\mathrm{Hz})$ & $1,02 \mathrm{E}+07$ \\
\hline Sound Speed $(\mathrm{m} / \mathrm{s})$ & $2,25 \mathrm{E}+3$ \\
\hline Density $\left(\mathrm{kg} / \mathrm{m}^{3}\right)$ & $1,47 \mathrm{E}+3$ \\
\hline Acoustic Impedance $Z\left[10^{6} \mathrm{~kg} / \mathrm{m}^{2} \mathrm{~s}\right]$ & 3.3075 \\
\hline Relative Dielectric constant $\varepsilon \mathrm{r}$ & 12 \\
\hline Piezoelectric Coefficient $\mathrm{d}_{33}(\mathrm{C} / \mathrm{N})$ & $3,40 \mathrm{E}-11$ \\
\hline Elastic compliance coefficient $S_{33}^{E}(1 / \mathrm{Pa})$ & $4,72 \mathrm{E}-10$ \\
\hline Max Applied Force/Excitation Tension $(\mathrm{N} / \mathrm{V})$ & $5,14 \mathrm{E}-02$ \\
\hline Max Operating Frequency Underwater $(\mathrm{Hz})$ & $1,04 \mathrm{E}+6$ \\
\hline Transmitted wave percentage at $1 \mathrm{MHz}(\%)$ & $78 \%$ \\
\hline
\end{tabular}

The emitter transducer was mounted in a structure with two PVDF layers of $110 \mu \mathrm{m}$ thickness with $20 \mathrm{~mm}$ diameter, where it was glued to a stainless steel mass and the outside surface was isolated with a thin silicone layer to protect it from water. The layers were connected in parallel to the excitation circuit, thus each layer is independently excited with the maximum voltage. By bonding reversed polarization layers, the electrodes between the glue have the same electrical potential and there is no current between them. This consequently reduces the power consumption once the parasite capacitance was disabled.

Fig. 1 shows the transducer frequency response and the power consumption in a $12 \mathrm{~m}$ distance with a $12 \mathrm{~V}$ excitation voltage.

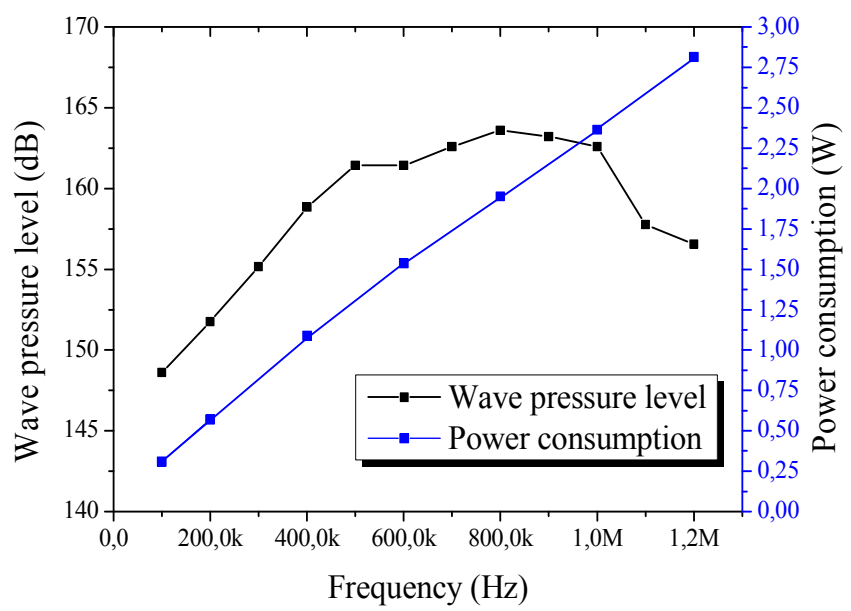

Fig. 1. Transducer frequency response and power consumption.

As it is possible to verify the transducer shows a flat response between $500 \mathrm{kHz}$ and $1 \mathrm{MHz}$ and only varies $5 \mathrm{~dB}$ between $100 \mathrm{kHz}$ and $500 \mathrm{kHz}$. The transducer also displays low power consumption at $1 \mathrm{MHz}$ since it only consumes $2.3 \mathrm{~W}$.

\section{SYSTEM DESCRIPTION}

From the developer point of view, the system is divided into three main layers, namely: application layer, hardware layer and physical layer, as it is presented in Fig. 2.

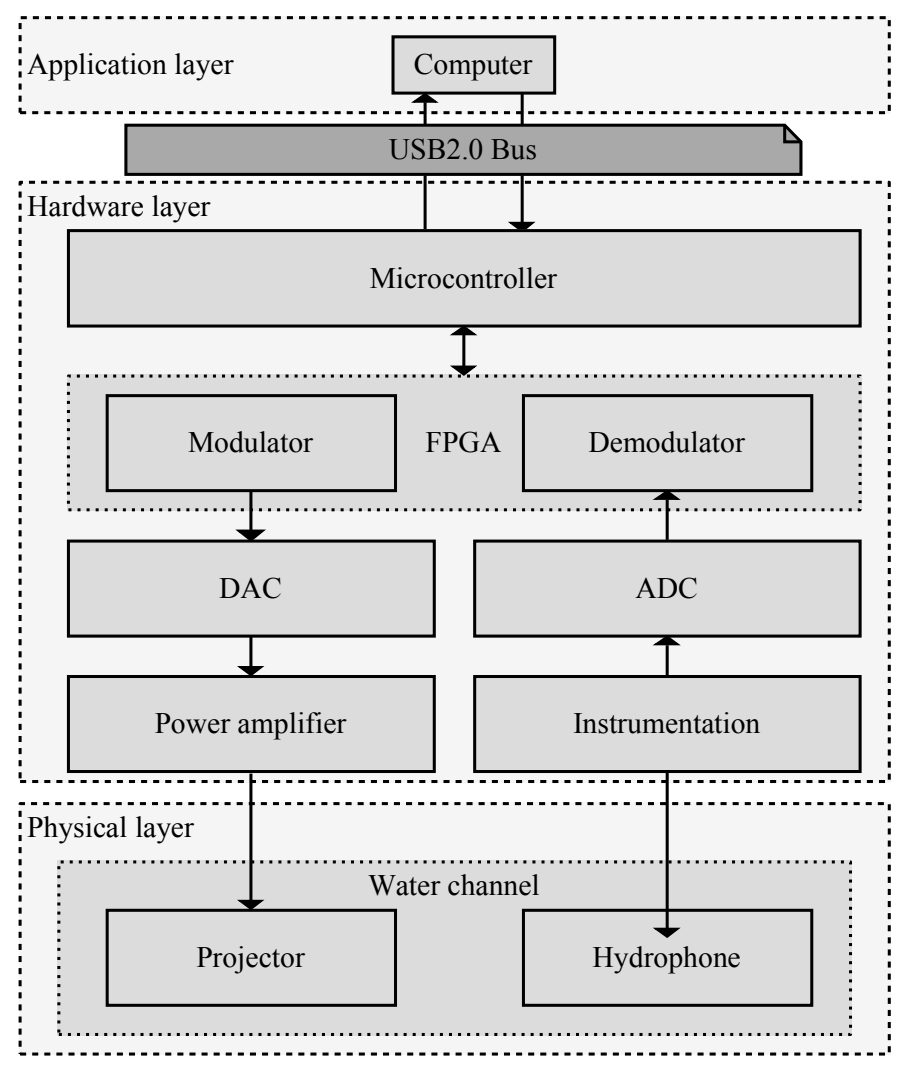

Fig. 2. System archietcture: application layer, hardware layer and physical layer. 
At the application layer the system performs the user interface, the data processing and retrieving process and it handles the communications with the hardware layer.

The hardware layer responds to the application layer in a slave mode. At this layer, the data coming from the application is decoded and modulated and amplified in voltage and power to be sent to the physical layer. In a similar way, the data coming from the physical layer is acquired, demodulated and sent to the application. The hardware layer is described in more detail in the section 4 .

The physical layer is basically made of transceivers, the emitter and receiver, and the water channel itself.

The hardware connection between the three layers was chosen based on the data types been exchanged and the expected symbol rate. Therefore, the connection between the computer, where the application layer is implemented and the acoustic modem, which is part of the hardware layer, should be USB.

The decision to use a USB connection is based on the fact that this connection protocol enables high bandwidth, $480 \mathrm{Mb} / \mathrm{s}$ in case of USB2.0, which is enough for this project's requirements, and presents also the advantage of being the most used connector in computers and mobile devices, which meets other requirements.

\section{PHYSICAL LAYER}

The emitter transducer used was a homemade PVDF piston type transducer with $20 \mathrm{~mm}$ diameter and a thickness with $2 \times 110 \mu \mathrm{m}$ structure. The selected hydrophone was a Cetacean Research $^{\mathrm{TM}}$ C304XR hydrophone with an effective sensibility of $-181 \mathrm{~dB}, 1 \mathrm{~V} / \mu \mathrm{Pa}$, a linear frequency range $( \pm 3 \mathrm{~dB})$ between 0.012 and $1000 \mathrm{kHz}$ and a frequency range $(+3 /-12 \mathrm{~dB})$ between 0.005 and $2000 \mathrm{kHz}$ [11].

\section{Reprogrammable ACOUSTIC MODEM}

At the hardware level, the signal path is divided into output signal (going out the modulator) and an input signal (coming into the demodulator). Both the input and output signals exist in analog and digital forms.

The output signal enters the hardware layer at the microcontroller, through the USB2.0 bus. Then, the microcontroller feeds the modulator implemented in the FieldProgrammable Gate Array (FPGA) with one byte at a time. The modulator processes the received data and generates a 14 bit word to drive the DAC, which generates an analog signal. From this point on, in the signal path, the signal assumes the analog form. The generated analog signal is then amplified in voltage and power and applied to the ultrasonic projector which is part of the physical layer.

The input signal enters the hardware layer through the hydrophone being filtered, amplified and then gets sampled by the ADC. From this point on, in the input signal path, the signal is digital. After sampling the signal it is converted into a 14 bit word which is then passed to the demodulator that in turn feeds the microcontroller with a single byte at a time.
As the base for the hardware layer a ZTEX USB-FPGA Module $1.15 \mathrm{~d}$ was selected, provided with a microcontroller Cypress CY7C68013A EZ-USB FX2 and a Xilinx Spartan 6 FPGA. An instrumentation board was developed and mounted on this module as shown in Fig. 3.

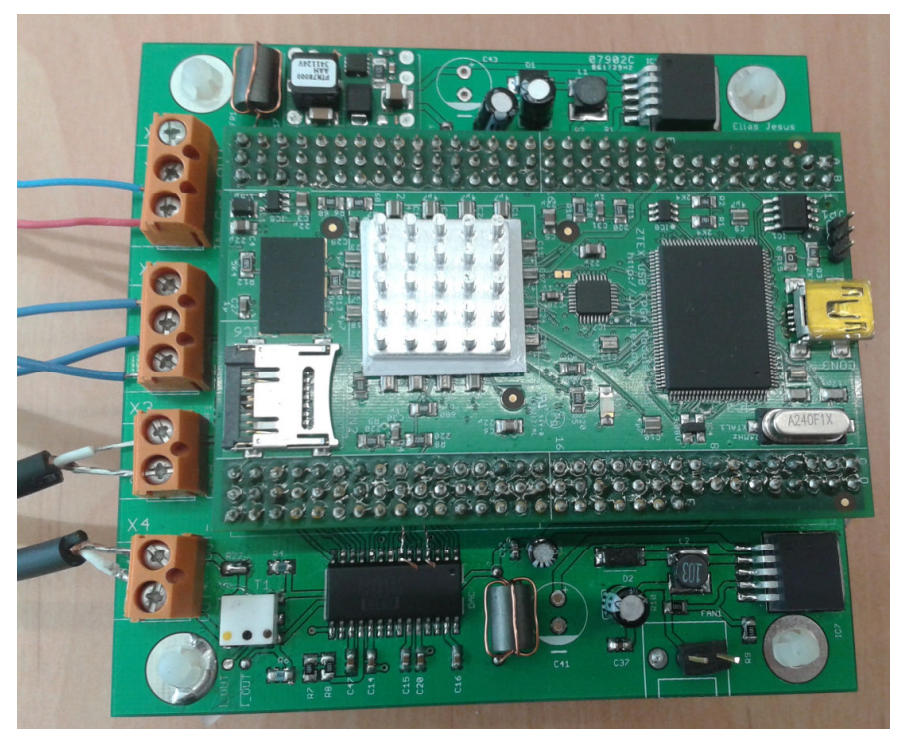

Fig. 3. System impelementation: control unit and instrumentation.

The instrumentation board is equipped with a High-Speed 14 bit ADC (AD9244) and DAC (DAC904) as well as an antialiasing LPF (LT6600-5) and a High-Speed ProgrammableGain Amplifier (THS7001).

The system is powered by $12 \mathrm{~V}$ batteries, so a Boost converter is required to transform the non-fixed input voltage into a fixed output voltage of $30 \mathrm{~V}$.

Because the system has to generate a sinusoidal signal with $30 \mathrm{~V}$ amplitude a negative voltage of $-30 \mathrm{~V}$ has to be created. To do so, instead of generating $-30 \mathrm{~V}$, an H-Bridge was used inverting the existing $30 \mathrm{~V}$ supply for the negative semi cycle.

To drive the $30 \mathrm{~V} \mathrm{H}$-Bridge a bipolar signal amplifier was used. This signal amplifier was built using operational amplifiers, which amplify signals in the range of $\pm 15 \mathrm{~V}$.

The analog signal enters the power electronics through a LPF to remove possible high frequency noise. It then goes through a RF transformer which acts as a HPF removing signals of frequencies bellow $100 \mathrm{kHz}$ and converting the single ended input to a differential output.

The differential signals, generated by the RF transformer, are $180^{\circ}$ out of phase from each other. These two signals are both amplified by an operational amplifier to drive the $\mathrm{H}$ Bridge. The final prototype is presented in Fig. 4. 


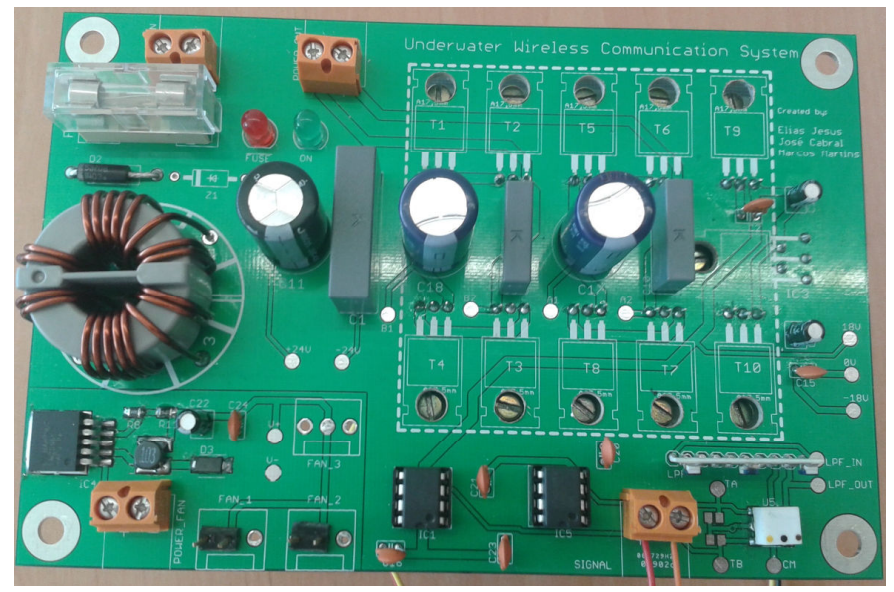

Fig. 4. H-Bridge Power Amplifier PCB Board.

The signal enters the LPF and then is transformed by the $\mathrm{RF}$ transformer. At the secondary of the transformer the middle output is connected to the ground which configures it to operate in differential mode. The RF transformer output signal is then amplified by the LM7171 which drives the push-pull.

An operational amplifier drives the power push-pull BJTs. The two BJTs are set in a Darlington configuration to increase its current sourcing capabilities. It also provides extra protection for the operational amplifier.

This hardware solution has the resources necessary for future re-designs of improved implementations. However, the system is built in modules which allow the improvement of a section without the replacement of the rest of the hardware.

\section{COMPUTER VISION - APPLICATION LAYER}

In the application layer a computer vision application was implemented where the image is compressed to fit in the available data rate attempting to compromise the image quality as little as possible.

In order to provide video streaming, two algorithms were developed based on the Open Source Computer Vision library also known as OpenCV. The flowcharts of Fig. 5 illustrate the algorithm steps for sending and receiving the video data.
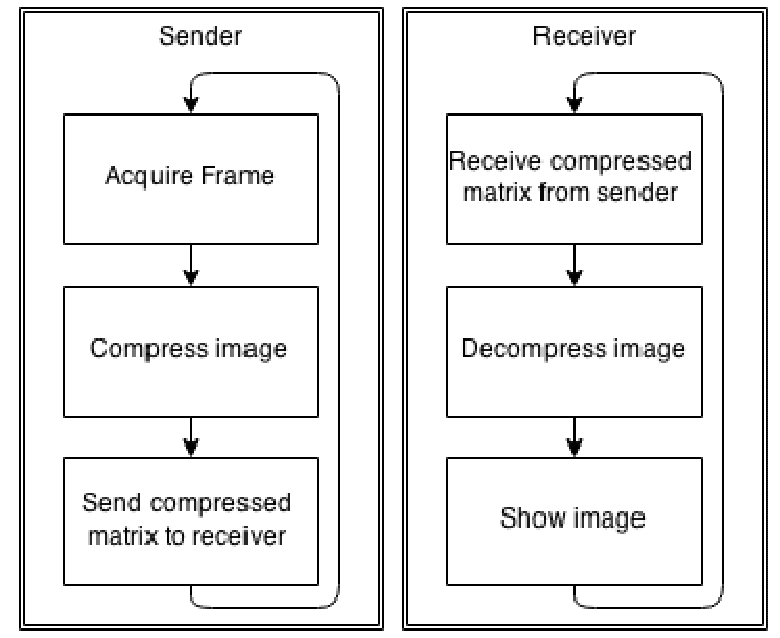

Fig. 5. Flowcharts of video sender (left) and video receiver (right).
On the sender side, a video frame is acquired from a camera and then compressed using the JPEG algorithm (cvEncodeImage function) provided by the OpenCV library. The compression quality is adjustable and it should provide the best compromise between image quality and compression ratio. This in turn allows different frame rates to be achieved. A higher compression ratio results in a lower quality image and hence in a higher frame rate (and vice-versa) as shown in Figure 6.

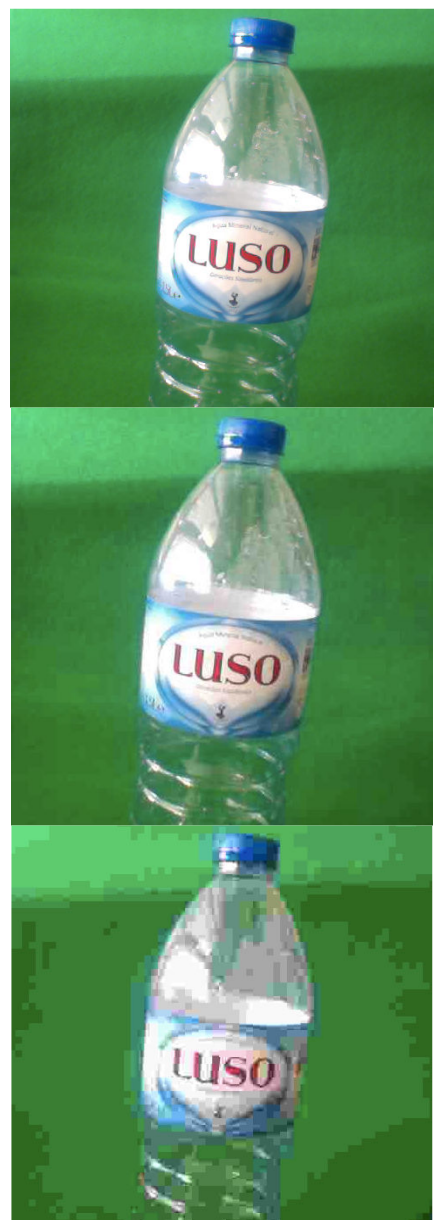

Fig. 6. Original captured image (top), 25\% quality (middle), 5\% quality (below).

The captured RGB image has a resolution of $640 \times 480$ pixels with 24 bit depth. When compressed to $25 \%$ quality in the JPEG encoder some loss in the color gradient can be found although the image can still be considered of good quality. On the other hand with $5 \%$ quality the image is visibly affected and the color gradient is completely lost. In both cases the achieved bit rate was close to $1 \mathrm{Mbps}$ with an increase of frames per second on the lower quality. Table 1 summarizes the resulting data from the images of Fig. 6 .

TABLE 2 - SUMMARY OF THE RESUlting DATA FROM THE IMAGES OF Fig. 6.

\begin{tabular}{|l|c|c|c|c|c|}
\hline $\begin{array}{l}\text { Original } \\
\text { size } \\
\text { (bytes) }\end{array}$ & $\begin{array}{l}\text { Compressed } \\
\text { JPQG matrix } \\
\text { (bytes) }\end{array}$ & $\begin{array}{l}\text { Compression } \\
\text { ratio }\end{array}$ & $\begin{array}{l}\text { Achieved } \\
\text { bit rate } \\
\text { (kbps) }\end{array}$ & $\begin{array}{l}\text { Frames } \\
\text { per } \\
\text { second }\end{array}$ & $\begin{array}{l}\text { JPEG } \\
\text { quality } \\
\text { (\%) }\end{array}$ \\
\hline $1,228,800$ & 10,014 & $1: 122$ & 926 & 12 & 25 \\
\hline $1,228,800$ & 6,550 & $1: 187$ & 973 & 19 & 5 \\
\hline
\end{tabular}


When compressing a frame, a matrix is created containing the compressed data from the JPEG algorithm. The sender must then send to the receiver the size of this matrix in order to allow the receiver to create a matrix of the same size. Then all the data from the compressed matrix is sent to the receiver via the water transducer. On the last word sent, the receiver passes this new matrix with the received data to the uncompressing function of the OpenCV library (cvDecodeImage function). This function returns an uncompressed image that is shown to the user on an OpenCV window.

At the moment the system is still under implementation, thus error checking and correction is not being enforced. Since it is a one-way only solution no back acknowledge is allowed. Therefore different approaches are being studied in order to implement a strong sending mechanism and a robust receiving decoder.

\section{EXPERIMENTAL TESTS AND RESULTS}

The system testing was divided in three parts. In the first part the acoustic modem was tested in order to analyze the communication performance. In the second part it is tested the relationship between the compression level, the image quality and frames per second in the streaming process, considering the limited data rate in the acoustic communication. The last part will be the integration of all system components and the quality of the video streaming will be measured in laboratory conditions (tank) and in the field (ocean, rivers).

\section{A. Acoustic Modem Test}

The experimental tests were carried out in a swimming pool with $12 \mathrm{~m}$ long, $4 \mathrm{~m}$ wide and $3 \mathrm{~m}$ deep. The measurements were performed with $12 \mathrm{~m}$ distance, $50 \mathrm{~cm}$ deep and in the middle of the pool ( $2 \mathrm{~m}$ either side). It was used fresh water at a temperature of $13{ }^{\circ} \mathrm{C}$ and with a $\mathrm{pH}$ of 7.2 .

The data rate test was performed in a half-duplex point-topoint communication using an OOK modulation with a $1 \mathrm{MHz}$ single carrier. The BER was measured for $1 \mathrm{Mbps}, 512 \mathrm{kbps}$ and $256 \mathrm{kbps}$ baud rates. The measurements were performed at two RS232 ports, allowing for sending and receiving the file on the same computer for comparison. The file size was $2 \mathrm{MB}$ long and no type of error control mechanisms was implemented. The results registered were $3 \times 10^{-3}$ BER with 1 Mbps, $2.3 \times 10^{-5}$ with $512 \mathrm{kbps}$ and $1 \times 10^{-8}$ with $256 \mathrm{kbps}$.

The elevated BER value was duo to the lack of control over the received data. In the BER measurement an error was detected in the demodulations which convert noise peaks in a trigger of a communication starting flag, resulting in receiving faulty absent data.

\section{B. Computer vision}

The conducted experiments tested different combinations of video resolution, video quality and frame rate and they revealed that video streaming was achieved with good quality at a resolution of $640 \times 480$ pixels attaining $12 \mathrm{fps}$. For each application the user can adjust one of these parameters (resolution and video quality) in order to obtain the intended frame rate. This compromise is always necessary but is somehow flexible as different compression ratios can be created depending on the captured scenario due to the JPEG compression algorithm operation. The tests also revealed that video streaming was achieved despite the limited data rate imposed by acoustic modem. However, without the proper mechanisms, error detection and correction for each byte lost means frame loss possibility.

\section{Streaming Video Results}

The streaming video tests are still ongoing. Although the application developed was a mechanism of self-regulation in order to maintain the video quality by varying the frame rate or maintain the frame rate by varying the video quality for data rate available.

\section{CONCLUSIONS}

In this work an underwater acoustic modem that allows communications over at least $20 \mathrm{~m}$, achieving a maximum data rate of $1 \mathrm{Mbps}$ with a $1 \mathrm{MHz}$ carrier is presented. The modem characteristics allow high quality real time video streaming. The presented system is based on a poly (vinylidene fluoride) PVDF ultrasonic emitter transducer which is capable of sending high quality signals but less powerful, comparing piezoceramic transducers, needed for digital modulations with high symbol rates per carrier period.

The maximum communication range is reduced by the increasing of the data rate even for the same carrier frequency, because with the increasing number of symbols per period the acoustic power of the received signal decreases.

The modem was tested for $1 \mathrm{Mbps}, 512 \mathrm{kbps}$ and $256 \mathrm{kbps}$ baud rates. The results registered were $3 \times 10^{-3}$ BER with 1 Mbps, $2.3 \times 10^{-5}$ with $512 \mathrm{kbps}$ and $1 \times 10^{-8}$ with $256 \mathrm{kbps}$.

The tests show that there is still room for modem improvements such as the implementation of multi-frequency modulations.

\section{ACKNOWLEDGMENT}

This work has been supported by FCT (Fundação para a Ciência e Tecnologia) in the scope of the project: PEstOE/EEA/UI04436/2015.

This work has been supported by FCT - Fundação para a Ciência e Tecnologia within the Project Scope: PestOE/EEI/UI0319/2014.

\section{REFERENCES}

[1] A. Caiti, K. Grythe, J. M. Hovem, S. M. Jesus, A. Lie, A. Munafo, T. A. Reinen, A. Silva, and F. Zabel, "Linking Acoustic Communications and Network Performance: Integration and Experimentation of an Underwater Acoustic Network," IEEE J. Ocean. Eng., vol. 38, no. 4, pp. 758-771, Oct. 2013.

[2] D.-G. for R. and I. European Commission, "Towards European integrated ocean observation," 2013.

[3] M. Chitre, S. Shahabudeen, and M. Stojanovic, "Underwater Acoustic Communications and Networking: Recent Advances and Future Challenges," Mar. Technol. Soc. J., vol. 42, no. 1, pp. 103116, Mar. 2008. 
[4] I. F. Akyildiz, D. Pompili, and T. Melodia, "Underwater acoustic sensor networks: research challenges," Ad Hoc Networks, vol. 3, no. 3, pp. 257-279, May 2005.

[5] X. Che, I. Wells, G. Dickers, P. Kear, and X. Gong, "Re-evaluation of RF electromagnetic communication in underwater sensor networks," IEEE Commun. Mag., vol. 48, no. 12, pp. 143-151, Dec. 2010.

[6] G. Baiden, Y. Bissiri, and A. Masoti, "Paving the way for a future underwater omni-directional wireless optical communication systems," Ocean Eng., vol. 36, no. 9-10, pp. 633-640, Jul. 2009.

[7] M. Stojanovic and J. Preisig, "Underwater acoustic communication channels: Propagation models and statistical characterization," IEEE Commun. Mag., vol. 47, no. 1, pp. 84-89, Jan. 2009.

[8] M. Stojanovic, J. Catipovic, and J. G. Proakis, "Adaptive multichannel combining and equalization for underwater acoustic communications," J. Acoust. Soc. Am., vol. 94, no. 3, p. 1621, 1993.

[9] S. I. and A. S. Sérgio M. Jesus, "Accounting for source depth variations in time-reversal communications," Submitt. to J. Acoust Soc. Am., 2014.

[10] S. J. J. Gomes, A. Silva, "Experimental assessment of time-reversed OFDM underwater communications," J. Acoust. Soc. Am., vol. 123, no. 5, p. 3891, 2008.

[11] M. Stojanovic, L. Freitag, and P. Willett, "Multicarrier Communication Over Underwater Acoustic Channels With
Nonuniform Doppler Shifts,” IEEE J. Ocean. Eng., vol. 33, no. 2, pp. 198-209, Apr. 2008.

[12] S. Roy, T. M. Duman, V. McDonald, and J. G. Proakis, "High-Rate Communication for Underwater Acoustic Channels Using Multiple Transmitters and Space-Time Coding: Receiver Structures and Experimental Results," IEEE J. Ocean. Eng., vol. 32, no. 3, pp. 663-688, Jul. 2007.

[13] E. M. Sözer and M. Stojanovic, "Reconfigurable acoustic modem for underwater sensor networks," Proc. 1st ACM Int. Work. Underw. networks - WUWNet '06, p. 101, 2006.

[14] N. Nowsheen, C. Benson, and M. Frater, "A high data-rate, software-defined underwater acoustic modem," in OCEANS 2010 MTS/IEEE SEATTLE, 2010, pp. 1-5.

[15] M. S. Martins, "Ultrasonic Wireless Broadband Communication System for Underwater Applications," PhD Thesis - Universidade do Minho, 2013.

[16] M. Martins, V. Correia, J. M. Cabral, S. Lanceros-Mendez, and J. G. Rocha, "Optimization of piezoelectric ultrasound emitter transducers for underwater communications," Sensors Actuators A Phys., vol. 184, pp. 141-148, Sep. 2012.

[17] M. S. Martins, N. Pinto, G. Rocha, S. L. Mendez, and J. Cabral, "Development of a 1 Mbps Low Power Acoustic Modem for Underwater Communications," in 2014 IEEE International Ultrasonics Symposium (IUS), 2014. 